\title{
A critical review of trials of transcranial direct current stimulation and trigeminal nerve stimulation for depression: the issue of treatment-emergent mania
}

\author{
Revisão crítica da literatura de ensaios clínicos em estimulação transcraniana \\ por corrente contínua e estimulação de nervo trigêmeo para depressão: \\ o problema da mania tratamento-emergente
}

Pedro Shiozawa, ${ }^{1}$ Quirino Cordeiro, ${ }^{1}$ Hyong Jin Cho, ${ }^{2}$ Alisson Paulino Trevizol, ${ }^{1}$ Elisa Brietzke ${ }^{3}$

\begin{abstract}
Objective: This study is a critical review analyzing occurrence of treatment-emergent mania (TEM) related to transcranial direct current stimulation (tDCS) and trigeminal nerve stimulation (TNS).

Method: We present a systematic review of the literature on TEM related to tDCS and TNS treatment for major depressive disorder (MDD), conducted in accordance with the recommendations from Cochrane Group and the PRISMA guidelines.

Results: Our search identified few reported episodes of TEM in the literature. In fact, we found 11 trials focused on treatment of MDD (seven controlled trials of tDCS and four trials of TNS, three open label and one controlled). We highlight the need for safety assessment in clinical research settings to establish with precision and in larger samples the risks inherent to the technique under investigation.

Conclusion: Safety assessment is of fundamental importance in clinical research. TEM is a very important safety issue in MDD trials. Further and larger controlled trials will help to clarify both the safety and the clinical effects of combinations of pharmacotherapy and TDCS or TNS in daily clinical practice.

Keywords: Neuromodulation, major depressive disorder, mania, transcranial direct current stimulation, trigeminal nerve stimulation.
\end{abstract}

Resumo

Objetivo: $O$ presente estudo consiste em uma revisão e análise crítica da ocorrência de mania tratamento-emergente (TEM) relacionada a estimulação transcraniana por corrente contínua (ETCC) e estimulação do nervo trigêmeo (TNS).

Método: Apresentamos uma revisão sistemática de literatura sobre TEM relacionada a ETCC e TNS no tratamento de transtorno depressivo maior (TDM), conduzida de acordo com as recomendações do Grupo Cochrane e protocolo PRISMA.

Resultados: A pesquisa identificou poucos relatos de TEM na literatura. Na verdade, foram encontrados 11 ensaios clínicos com foco no tratamento de TDM (sete estudos controlados de ETCC e quatro de TNS, sendo três abertos e um controlado). Destacamos a necessidade de avaliações de segurança em pesquisas clínicas para se estabelecer com maior precisão e em amostras maiores os riscos inerentes à técnica sob investigação.

Conclusão: Avaliação de segurança é fundamental na pesquisa clínica. A TEM é um efeito adverso importante no tratamento do TDM. Maiores ensaios clínicos controlados ajudarão a esclarecer os efeitos clínicos e a segurança da combinação de psicotrópicos e ETCC ou TNS.

Descritores: Neuromodulação, transtorno depressivo maior, mania, estimulação transcraniana por corrente contínua, estimulação de nervo trigêmeo.

\footnotetext{
${ }^{1}$ Centro Interdisciplinar de Neuromodulação Clínica, Faculdade de Ciências Médicas, Santa Casa de São Paulo, São Paulo, SP, Brazil. 2 University of California (UCLA), San Diego, USA. ${ }^{3}$ Laboratório Interdisciplinar de Neurociências Clínicas (LiNC), Departamento de Psiquiatria, Universidade Federal de São Paulo (UNIFESP), São Paulo, SP, Brazil.

Financial support: none.

Submitted Apr 19 2016, accepted for publication Jul 25 2016. No conflicts of interest declared concerning the publication of this article.

Suggested citation: Shiozawa P, Cordeiro Q, Cho HJ, Trevizol AP, Brietzke E. A critical review of trials of transcranial direct current stimulation and trigeminal nerve stimulation for depression: the issue of treatment-emergent mania. Trends Psychiatry Psychother. 2017;39(1):48-53. http://dx.doi.org/10.1590/22376089-2016-0027
} 


\section{Introduction}

There has been growing interest within Clinical Psychiatry in developing new strategies for treatment of major depressive disorder (MDD). This movement is founded on the possibility of overcoming current challenges to standard pharmacological therapy, such as low adherence rates, treatment resistance, and side effects. ${ }^{1-3}$

Non-invasive brain stimulation (NIBS) techniques appear to fit with this current scientific trend. In fact, for depressive disorder, electroconvulsive therapy (ECT) is the longest-standing psychiatric treatment available and it has shown solid results in terms of ameliorating clinical symptoms. However, given both the technical challenges and high cost, use of ECT has been overtaken by certain pharmacological approaches in daily practice. ${ }^{4,5}$ Neuromodulation techniques include novel procedures such as transcranial direct current stimulation (tDCS) and trigeminal nerve stimulation (TNS). In tDCS, the anode increases and the cathode decreases cortical excitability of the areas beneath them. ${ }^{6}$ In turn, TNS is an incipient interventional strategy based on application of an electric current over a branch of the trigeminal nerve with further propagation of the stimuli towards brain areas related to psychiatric symptoms. ${ }^{7}$

Some of the general characteristics of these techniques that have attracted the scientific community's attention are their low cost, portability, and ease of use. Several authors have evaluated their clinical applications in Psychiatry, notably to treat individuals suffering from MDD. However, clinical studies of tDCS and other NIBS techniques for treating MDD have reported some fundamental issues, such as treatment-related adverse effects, including treatment-emergent mania (TEM). Since the gold standard treatment for mood disorders is currently pharmacotherapy, most reports of TEM have been related to antidepressant, ${ }^{8}$ mood stabilizers, ${ }^{9}$ and other add-on medications. ${ }^{10,11}$ More recently, with the growing interest in NIBS, recent data has also linked these new approaches to TEM.

All new techniques must be carefully assessed before their inclusion in the therapeutic arsenal, even if preliminary results are favorable. The objective of this study was to conduct a systematic literature review of TEM in clinical trials of tDCS and TNS for MDD.

\section{Methods}

A review of the literature on TEM related to tDCS and TNS was conducted in two stages, as follows:
1) we looked for case reports describing emergence of hypomanic/manic symptoms after tDCS or TNS treatment protocols; 2) we examined reports of mania/hypomania in tDCS or TNS randomized clinical trials. Both analyses were conducted according to recommendations from Cochrane Group and the PRISMA guidelines. ${ }^{12}$ Two authors (PS and QC) performed independent systematic reviews and data extraction, and any discrepancies were resolved by consensus.

We reviewed the following references and databases:

(a) MEDLINE and EMBASE databases using the keywords: 1) "transcranial stimulation"; 2) "tDCS"; 3) "brain polarization"; 4) "electric stimulation"; 5) "electric polarization"; 6) "non-invasive brain stimulation"; 7) "NIBS"; 8) "depressive disorder"; 9) "depression"; 10) "depressive episode"; 11) "mania"; 12) "hypomania." The Boolean terms were input as follows: [(1) OR (2) OR (3) OR (4) OR (5) OR (6) OR (7)] AND [(8) OR (9) OR (10) OR (11) OR (12)]. The search was performed up to March 3, 2015.

(b) Study references in articles and reviews identified in (a).

We also looked for controlled trials by contacting specialists in the field and by searching the website clinicaltrials.gov for additional unpublished/ongoing trials.

We adopted the following inclusion criteria: 1) manuscript written in English, Spanish, or Portuguese (in fact all articles identified were written in English); 2) randomized, sham-controlled trials; (for both TNS and tDCS studies); 3) open label trials for TNS (given the incipient use of TNS in the current research scenario). We excluded studies assessing conditions other than MDD or without reference to mania/hypomania symptoms as well as interventions other than tDCS or TNS (Figure 1).

"Database search on MEDLINE and Embase databases using the key words:
1) "transcranial stimulation"; 2) "tDCS"; 3) "brain polarization"; 4) "electric
stimulation"; 5) "electric polarization"; 6) "non-invasive brain stimulation"; 7)
"NIBS"; 8) "depressive disorder"; 9) "depression"; 10) "depressive episode";
11) "mania"; 12) "hypomania." The Boolean terms were input as follows: [(1)
OR (2) OR (3) OR (4) OR (5) OR (6) OR (7)] AND [(8) OR (9) OR (10) OR (11)
OR (12)]. The search was performed up to March 03, 2015."

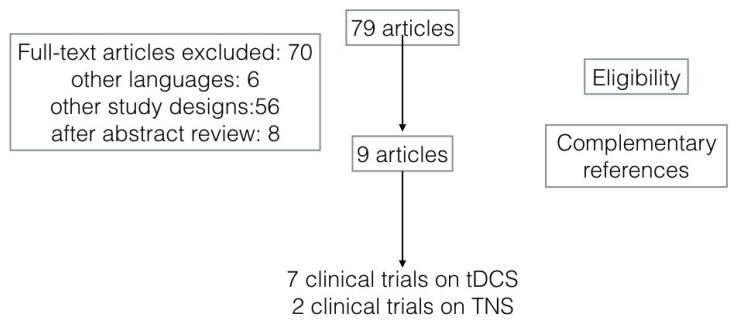

Figure 1 - Quorum assessment for eligible studies. 


\section{Results}

Our search yielded 11 trials focused on treatment of MDD (seven controlled trials of tDCS and four trials of TNS, three open labels and one controlled trial).

\section{Trigeminal nerve stimulation}

Our search returned three open and one controlled trial of TNS for MDD. Shiozawa et al. ${ }^{13}$ evaluated a total of 11 patients, with a mean age of 50.36 years (standard deviation $[S D]=11.8$ years; range: 30 to 60 years). Only one patient was male. Regarding depressive symptoms, there was a mean reduction of 5.72 points $(27.6 \%)$ on the Hamilton Depression Rating Scale-17 items (HDRS17) $(\mathrm{SD}=2.24$ points $)(p<0.001)$. Reductions of at least $50 \%$ were reported for all patients' scores. Only one patient did not attain scores indicative of remission (defined as HDRS-17 lower than 8). In two other openlabel trials 14,15 the authors reported the effects of TNS in 11 and five adults, respectively, in an 8-week openlabel outpatient trial. There was clinical amelioration of depressive symptoms $(p<0.01)$ and no adverse effects were observed, including TEM. The randomized controlled trial ( $n=40$; mean age of 47.50 years, SD $=12.05$ years) did not report any cases of TEM either. ${ }^{16}$

The authors of the TNS trials found no cases of TEM. However, manic symptoms were assessed by clinicallybased interviews, rather than using a structured assessment scale.

\section{Transcranial direct current stimulation}

Seven randomized controlled clinical trials assessed the effects of tDCS on the severity of MDD depressive symptoms ( $n=319$ patients; mean age of 43.62 years, SD $=10.00) \cdot 17-23$ In these studies, 10 cases of emergence of (hypo)manic symptoms were reported in three trials: $9(5.38 \%$ ) in active tDCS groups (all patients but one were on add-on therapy with antidepressants) and $1(0.65 \%)$ in a sham stimulation group (although this patient was also on add-on therapy with sertraline). There was an overall incidence of $3.13 \%$ for all patients on tDCS protocols studied.

Tables 1 and 2 show an overview of the studies selected.

Table 1 - Overview of randomized clinical trials for tDCS

\begin{tabular}{|c|c|c|c|c|c|c|c|c|c|c|c|}
\hline Study & Year & $\begin{array}{c}\mathbf{n} \\
\text { (active/ } \\
\text { sham) }\end{array}$ & $\begin{array}{c}\text { Mean age } \\
(\text { SD) }\end{array}$ & $\begin{array}{l}\text { Sex } \\
(\% f)\end{array}$ & $\begin{array}{c}\text { Current } \\
(\mathrm{mA})\end{array}$ & $\begin{array}{c}\text { Duration } \\
(\mathrm{min})\end{array}$ & Anode & Cathode & $\begin{array}{c}\text { No. } \\
\text { sessions }\end{array}$ & $\begin{array}{l}\text { Density } \\
\left(m^{2} / A\right)\end{array}$ & $\begin{array}{c}\text { (Hypo)mania } \\
\text { total (active/ } \\
\text { sham) }\end{array}$ \\
\hline $\begin{array}{l}\text { Boggio et } \\
\text { al. }^{21}\end{array}$ & 2008 & $21 / 10$ & $49(7)$ & 67.5 & 2 & 20 & F3 & Right supraorbital & 10 & 0.57 & $0 / 0$ \\
\hline Loo et al. ${ }^{18}$ & 2010 & $20 / 20$ & 49 (NR) & 55 & 1 & 20 & F3 & Right supraorbital & 5 & 0.28 & $1 / 0$ \\
\hline Loo et al. ${ }^{19}$ & 2012 & $33 / 31$ & $47.2(12.5)$ & 46.6 & 2 & 20 & F3 & Right supraorbital & 15 & 0.57 & $1 / 0$ \\
\hline Palm et al. ${ }^{20}$ & 2011 & $11 / 11$ & $57(12)$ & 50 & 1 or 2 & 20 & F3 & Right supraorbital & 10 & $0.28 / 0.57$ & $0 / 0$ \\
\hline $\begin{array}{l}\text { Blumberger } \\
\text { et al. } .^{17}\end{array}$ & 2012 & $13 / 11$ & $42.7(11.6)$ & 45.6 & 2 & 20 & F3 & $\mathrm{F} 4$ & 15 & 0.57 & $0 / 0$ \\
\hline $\begin{array}{l}\text { Fregni et } \\
\text { al. }{ }^{22}\end{array}$ & 2009 & $9 / 9$ & $48.2(10)$ & NR & 1 & 20 & F3 & Right supraorbital & 5 & 0.28 & $0 / 0$ \\
\hline $\begin{array}{l}\text { Brunoni et } \\
\text { al. }{ }^{23}\end{array}$ & 2013 & $60 / 60$ & $42(12)$ & 82 & 2 & 30 & F3 & $\mathrm{F} 4$ & 12 & 0.80 & $7 / 1$ \\
\hline
\end{tabular}

$\mathrm{NR}=$ not reported $; \mathrm{SD}=$ standard deviation; tDCS = transcranial direct current stimulation.

Table 2 - Overview TNS studies

\begin{tabular}{|c|c|c|c|c|c|c|}
\hline Study & Year & Design & Mean age (SD) & $\begin{array}{l}\text { Sex } \\
(\%)\end{array}$ & Condition studied & (Hypo)mania total \\
\hline Schrader et al. ${ }^{15}$ & 2011 & Open $(n=5)$ & $49.6(10.9)$ & 60 & MDD & no \\
\hline Cook et al. ${ }^{14}$ & 2013 & $\begin{array}{c}\text { Open-label }(n= \\
11)\end{array}$ & $48.1(8.3)$ & 63 & MDD & no \\
\hline Shiozawa et al. ${ }^{7}$ & 2014 & Open $(n=11)$ & $50.36(11.8)$ & 90 & MDD & no \\
\hline Shiozawa et al. ${ }^{16}$ & 2015 & RCT $(n=40)$ & $48.7(12.5)$ & 65 & MDD & no \\
\hline
\end{tabular}

MDD = major depressive disorder; RCT = randomized clinical trial; SD = standard deviation; TNS = trigeminal nerve stimulation . 


\section{Methodological considerations}

\section{Parameters}

The tDCS protocols that reported TEM were not uniform. Brunoni et al. ${ }^{23}$ performed a factorial study ( $n$ $=120$ ) with six weeks of treatment with 2-mA anodal left/cathodal right prefrontal tDCS (12 30-minute sessions: 10 consecutive sessions once daily on weekdays plus 2 extra sessions every other week). Loo et al.18 performed a double-blinded, randomized study with 40 participants with the following tDCS protocol: $1 \mathrm{~mA}$ current strength, five sessions, active or sham stimulation, delivery on alternate days. Anodal stimulation was centered over the left dorsolateral prefrontal cortex, with the cathode placed on the lateral aspect of the contralateral orbit. Finally, Loo \& Martin24 evaluated 64 participants with current depression. The participants received active or sham anodal tDCS to the left prefrontal cortex ( $2 \mathrm{~mA}, 15$ sessions over 3 weeks), followed by a 3-week open-label active treatment phase. Considering all the studies with tDCS, we found that the protocols were not the same. Most authors reported the use of $2 \mathrm{~mA}$ with 20-minute sessions. All cases were stimulated with anode placed over F3, while cathode was placed either over F4, right supraorbital, or extra-cephalically (in one case). The number of sessions was also variable from one study to another, varying from 5 to 15 sessions.

The TNS protocols used across different studies delivered similar signals, but employed somewhat different technologies and protocols. The common elements were a stimulation frequency of $120 \mathrm{~Hz}$, pulse duration of $250 \mu \mathrm{s}$, and cycling with $30 \mathrm{~s}$ on/30 s off. Two studies by a Brazilian group ${ }^{13,16}$ reported using an external stimulator (Neurodyn, Ibramed, Amparo, $\mathrm{SP}$, Brazil) and $25 \mathrm{~cm}^{2}$ electrodes consisting of salinesolution-soaked sponges positioned over supraorbital foramen for the 30 minute treatment period, during the day. Two studies by a US-based group ${ }^{14,15}$ reported using an external stimulator (EMS7500 Stimulator, TENS Products, Inc., Granby, CO, USA) and used a self-adhering gel electrode for an eight-hour treatment period at night, largely during sleep.

\section{Concurrent use of medication}

All but one of the patients who presented with TEM during the treatment were on pharmacotherapy, i.e., tDCS was used mainly as an add-on strategy. The same was observed for the case reports, where all subjects were on antidepressant protocols. The patients on the TNS trials were also on pharmacological treatment. Because of this, it is not possible to exclude the possibility that antidepressants were completely or partially responsible for TEM.

\section{Discussion}

The results of this review indicate that new neuromodulation methods such as tDCS and TNS are associated with a relatively low risk of TEM. The neurobiological basis of manic symptoms seems to be related to abnormal activity of the right dorsolateral prefrontal cortex (DLPFC). In fact, mania may exhibit hypofunction of the right DLPFC and contralateral hyperactivity. ${ }^{25,26}$ It is possible that TEM might also involve prefrontal malfunctioning, similar to the cortical impairment exhibited in mania. ${ }^{27}$

Following the rationale of prefrontal dysfunction, a recent case study found that anodal tDCS of the right dorsolateral prefrontal cortex ameliorated acute mania symptoms. ${ }^{27}$ More recently, another group published the pilot phase of a controlled trial of tDCS for bipolar depression. A total of five patients received active tDCS, two of whom exhibited clinical responses. The tDCS protocol consisted of 12 anodal-left/cathodal-right $2 \mathrm{~mA}$ tDCS sessions over the dorsolateral prefrontal cortex. ${ }^{28}$ Galvez et al. reported a case of a 33-year-old female with bipolar II disorder, who presented with TEM following a frontoextracephalic tDCS protocol for MDD. The patient had previously undergone treatment with a bifrontal electrode montage without exhibiting manic symptoms. ${ }^{29}$

Several research groups have focused on safety issues of tDCS for MDD. In fact, there is growing evidence related to manic shift and other safety considerations in MDD trials. Pooled results from different tDCS protocols will help to determine the optimal technical parameters for future trials. ${ }^{30,31}$

\section{TEM and current antidepressant therapy}

Antidepressants are nowadays the standard treatment option for major depression. However, the evidence is not so robust with relation to their use for bipolar depression. ${ }^{32,33}$ Antidepressants can precipitate TEM. In fact, about $40 \%$ of patients enrolled on a recent clinical trial assessing bipolar depression (STEP-BD trial) self-reported manic or hypomanic switch associated with antidepressant use. ${ }^{34}$ Moreover, Bottlender et al. ${ }^{1}$ also evaluated the occurrence of TEM in 158 bipolar I inpatients. Compared with patients who did not switch $(n=119)$, those who developed TEM $(n=39)$ had a significantly higher rate of antidepressant use (51 vs. $80 \%$ ). The same group reported that 3-month adjunctive 
antidepressant treatment not only did not speed up time to recovery from an index episode of bipolar depression compared with no antidepressant treatment, but was also associated with significant manic symptoms at study endpoint. ${ }^{35}$

Several different studies focused on pharmacotherapy have assessed TEM. ${ }^{36,37}$ With relation to the differences in TEM rates in unipolar/bipolar depression, Benazzi ${ }^{36}$ showed that bipolar patients had a significantly greater risk of developing TEM than unipolar depression patients (17.3 vs. $5.8 \%$ ). As for the pharmacological drug class, another group ${ }^{37}$ demonstrated that the rate of TEM is about $1 \%$ for unipolar depression patients, irrespective of antidepressant pharmacological class, whereas for bipolar depression, manic switch occurs substantially more often with tricyclic agents $(11.2 \%)$ than with selective serotonin reuptake inhibitors (3.7\%) or placebo $(4.2 \%)$. In the trials analyzed here, patients were selected based on clinical MDD diagnoses, i.e., the risk of developing TEM could not be related to previous bipolar diagnoses. In clinical research into MDD, the occurrence of (hypo)manic episodes is a challenging issue due to lack of a significant predictor that has been systematically addressed to date.

The results of this study should be interpreted in the light of some limitations. Given the still incipient nature of clinical research into tDCS for MDD, patients with a high risk for TEM (refractory, severe depression, psychiatric comorbidities) were more likely to be placed on to some tDCS interventional protocols. Another issue of concern is that, regardless of differences in protocols, all reported cases of TEM with tDCS shared the factor of concomitant use of antidepressant drugs, i.e., tDCS protocols were used as an add-on therapy for these patients. Because of this, we can hypothesize that TEM could be more attributable to the combination antidepressant + tDCS than to tDCS per se. Therefore, the present prevalence of TEM among tDCS studies might be influenced by interactions between these interventions and so the attribution of TEM to tDCS should be interpreted with caution. Finally, TNS has not hitherto been linked to TEM, although only one controlled clinical trial has been published evaluating TNS for MDD to date and no specific screening scale was used to evaluate mania during follow up.

\section{TEM and neuromodulation approaches: what about reporting bias?}

Some authors have already emphasized the occurrence of TEM following tDCS interventions and the importance of medical care during follow up. ${ }^{38}$ Clinical data suggest that the occurrence of manic symptoms related to tDCS tends to be more likely than with other neuromodulation strategies, similar to antidepressants. ${ }^{23}$ In fact, the rate of TEM occurrence associated with transcranial magnetic stimulation protocols (rTMS) in a pooled sample of both unipolar and bipolar disorders was $0.84 \%$ for the active rTMS treatment group and $0.73 \%$ for the sham group. ${ }^{39}$ The rate of TEM with tDCS was significantly higher than for TMS. We believe this data may be jeopardized by significant differences between studies of the two strategies in terms of clinical effects and sample sizes. However, further trials will help to provide more robust evidence on which to base this important discussion.

Indeed, in a previous systematic review, the authors identified a selective reporting bias affecting reporting, assessing, and publishing of adverse effects related to tDCS. A revised adverse effects questionnaire was proposed for assessments in tDCS studies in order to improve systematic reporting of such events. ${ }^{38}$ For example, severity of manic symptoms at baseline is a well-known predictor of TEM. ${ }^{40}$ However, several trials did not include a systematic assessment of manic symptoms at baseline. Incorporation of the new diagnosis of mixed depression, as proposed in the Diagnostic and Statistical Manual of Mental Disorders, 5th edition (DSM-5), will probably refine our understanding of TEM and improve our knowledge about the neurobiology of switch and cycling.

\section{Conclusion}

Safety assessment is of fundamental importance in clinical research. TEM is a very important safety issue in MDD trials. Further and larger controlled trials will help to clarify both the safety and the clinical effects of combinations of pharmacotherapy and tDCS or TNS in daily clinical practice.

\section{References}

1. Bottlender R, Sato T, Kleindienst N, Strauss A, Moller HJ. Mixed depressive features predict maniform switch during treatment of depression in bipolar I disorder. J Affect Disord. 2004;78:149-52.

2. Anderson C, Roy T. Patient experiences of taking antidepressants for depression: a secondary qualitative analysis. Res Social Adm Pharm. 2013;9:884-902.

3. Montgomery SA. Why do we need new and better antidepressants? Int Clin Psychopharmacol. 2006;21:S1-S10.

4. Micallef-Trigona $B$, Spiteri J. Maintenance electroconvulsive therapy in a patient with treatment-resistant paranoid schizophrenia and comorbid epilepsy. Case Rep Psychiatry. 2012;2012:374752.

5. Leclerc E, Mansur RB, Brietzke E. Determinants of adherence to treatment in bipolar disorder: a comprehensive review. J Affect Disord. 2013;149:247-52.

6. Nitsche MA, Cohen LG, Wassermann EM, Priori A, Lang N, Antal $A$, et al. Transcranial direct current stimulation: state of the art 
2008. Brain Stimul. 2008;1:206-23.

7. Shiozawa $P$, Silva ME, Carvalho TC, Cordeiro Q, Brunoni AR, Fregni $F$. Transcutaneous vagus and trigeminal nerve stimulation for neuropsychiatric disorders: a systematic review. Arq Neuropsiquiatr. 2014;72:542-7.

8. Post RM, Altshuler LL, Leverich GS, Frye MA, Nolen WA, Kupka RW, et al. Mood switch in bipolar depression: comparison of adjunctive venlafaxine, bupropion and sertraline. $\mathrm{Br}$ J Psychiatry. 2006; 189:124-31.

9. Bowden CL. Improving identification of treatment effectiveness in bipolar disorders. Bipolar Disord. 2003;5:77-8.

10. Calabrese JR, Bowden CL, Sachs G, Yatham LN, Behnke K Mehtonen $\mathrm{OP}$, et al. A placebo-controlled 18-month trial of lamotrigine and lithium maintenance treatment in recently depressed patients with bipolar I disorder. J Clin Psychiatry. 2003;64:1013-24.

11. Gao K, Yuan C, Wu R, Chen J, Wang Z, Fang Y, et al. Important clinical features of atypical antipsychotics in acute bipolar depression that inform routine clinical care: a review of pivotal studies with number needed to treat. Neurosci Bull. 2015;31:57288 .

12. Moher D, Liberati A, Tetzlaff J, Altman DG; PRISMA Group. Preferred reporting items for systematic reviews and metaanalyses: the PRISMA statement. PLoS Med. 2009;6:e1000097.

13. Shiozawa $P$, Duailibi MS, da Silva ME, Cordeiro Q. Trigeminal nerve stimulation (TNS) protocol for treating major depression: an open-label proof-of-concept trial. Epilepsy Behav. 2014;39:69.

14. Cook IA, Schrader LM, Degiorgio CM, Miller PR, Maremont ER, Leuchter AF. Trigeminal nerve stimulation in major depressive disorder: acute outcomes in an open pilot study. Epilepsy Behav. 2013;28:221-6.

15. Schrader LM, Cook IA, Miller PR, Maremont ER, DeGiorgio CM. Trigeminal nerve stimulation in major depressive disorder: first proof of concept in an open pilot trial. Epilepsy Behav. 2011;22:475-8.

16. Shiozawa P, da Silva ME, Netto GT, Taiar I, Cordeiro Q. Effect of a 10-day trigeminal nerve stimulation (TNS) protocol for treating major depressive disorder: a phase II, sham-controlled, randomized clinical trial. Epilepsy Behav. 2015;44:23-6.

17. Blumberger DM, Tran LC, Fitzgerald PB, Hoy KE, Daskalakis ZJ. A randomized double-blind sham-controlled study of transcranial direct current stimulation for treatment-resistant major depression. Front Psychiatry. 2012;3:74.

18. Loo CK, Sachdev P, Martin D, Pigot M, Alonzo A, Malhi GS, et al. A double-blind, sham-controlled trial of transcranial direct current stimulation for the treatment of depression. Int J Neuropsychopharmacol. 2010;13:61-9.

19. Loo CK, Alonzo A, Martin D, Mitchell PB, Galvez V, Sachdev P. Transcranial direct current stimulation for depression: 3-week, randomised, sham-controlled trial. Br J Psychiatry. 2012;200:529.

20. Palm U, Schiller C, Fintescu Z, Obermeier M, Keeser D, Reisinger $\mathrm{E}$, et al. Transcranial direct current stimulation in treatment resistant depression: a randomized double-blind, placebocontrolled study. Brain Stimul. 2012;5:242-51.

21. Boggio PS, Rigonatti SP, Ribeiro RB, Myczkowski ML, Nitsche MA, Pascual-Leone $A$, et al. A randomized, double-blind clinical trial on the efficacy of cortical direct current stimulation for the treatment of major depression. Int J Neuropsychopharmacol. 2008;11:24954.

22. Fregni F, Boggio PS, Nitsche MA, Marcolin MA, Rigonatti SP, Pascual-Leone A. Treatment of major depression with transcranial direct current stimulation. Bipolar Disord. 2006;8:203-4.

23. Brunoni $A R$, Valiengo $L$, Baccaro $A$, Zanão TA, de Oliveira JF, Goulart A, et al. The sertraline vs. electrical current therapy for treating depression clinical study: results from a factorial, randomized, controlled trial. JAMA Psychiatry. 2013;70:383-91.

24. Loo CK, Martin DM. Could transcranial direct current stimulation have unexpected additional benefits in the treatment of depressed patients? Expert Rev Neurother. 2012;12:751-3.

25. Michael N, Erfurth A, Pfleiderer B. Elevated metabolites within dorsolateral prefrontal cortex in rapid cycling bipolar disorder. Psychiatry Res. 2009;172:78-81.

26. Frey $B N$, Folgierini $M$, Nicoletti $M$, Machado-Vieira $R$, Stanley JA, Soares JC, et al. A proton magnetic resonance spectroscopy investigation of the dorsolateral prefrontal cortex in acute mania. Hum Psychopharmacol. 2005;20:133-9.

27. Galvez V, Alonzo A, Martin D, Mitchell PB, Sachdev P, Loo CK. Hypomania induction in a patient with bipolar II disorder by transcranial direct current stimulation (tDCS). J ECT. 2011;27:256-8

28. Pereira Junior Bde S, Tortella G, Tortella G, Lafer B, Nunes P, Benseñor IM, et al. The bipolar depression electrical treatment trial (BETTER): design, rationale, and objectives of a randomized, sham-controlled trial and data from the pilot study phase. Neural Plast. 2015;2015:684025. doi: 10.1155/2015/684025. Epub 2015 Mar 24.

29. Schestatsky $P$, Janovik N, Lobato MI, Belmonte-de-Abreu $P$, Schestatsky S, Shiozawa $\mathrm{P}$, et al. Rapid therapeutic response to anodal tDCS of right dorsolateral prefrontal cortex in acute mania. Brain Stimul. 2013;6:701-3.

30. Dell'Osso B, Priori A, Altamura AC. Efficacy and safety of transcranial direct current stimulation in major depression. Biol Psychiatry. 2011;69:e23-4.

31. Martin DM, Alonzo A, Mitchell PB, Sachdev P, Galvez V, Loo CK. Fronto-extracephalic transcranial direct current stimulation as a treatment for major depression: an open-label pilot study. J Affect Disord. 2011;134:459-63.

32. Jann MW. Diagnosis and treatment of bipolar disorders in adults: a review of the evidence on pharmacologic treatments. Am Health Drug Benefits. 2014;7:489-99.

33. McInerney SJ, Kennedy SH. Review of evidence for use of antidepressants in bipolar depression. Prim Care Companion CNS Disord. 2014;16. doi: 10.4088/PCC.14r01653. eCollection 2014.

34. Truman CJ, Goldberg JF, Ghaemi SN, Baldassano CF, Wisniewski SR, Dennehy EB, et al. Self-reported history of manic/hypomanic switch associated with antidepressant use: data from the Systematic Treatment Enhancement Program for Bipolar Disorder (STEP-BD). J Clin Psychiatry. 2007;68:1472-9.

35. Goldberg JF, Brooks JO 3rd, Kurita K, Hoblyn JC, Ghaemi SN, Perlis $\mathrm{RH}_{\text {, et }}$ al. Depressive illness burden associated with complex polypharmacy in patients with bipolar disorder: findings from the STEP-BD. J Clin Psychiatry. 2009;70:155-62.

36. Benazzi F. Antidepressant-associated hypomania in outpatient depression: a 203-case study in private practice. J Affect Disord. 1997;46:73-7

37. Peet $M$. Induction of mania with selective serotonin re-uptake inhibitors and tricyclic antidepressants. $\mathrm{Br} J$ Psychiatry. 1994; $164: 549-50$

38. Brunoni AR, Amadera J, Berbel B, Volz MS, Rizzerio BG, Fregni F. A systematic review on reporting and assessment of adverse effects associated with transcranial direct current stimulation. Int J Neuropsychopharmacol. 2011;14:1133-45.

39. Xia G, Gajwani P, Muzina DJ, Kemp DE, Gao K, Ganocy SJ, et al. Treatment-emergent mania in unipolar and bipolar depression: focus on repetitive transcranial magnetic stimulation. Int ] Neuropsychopharmacol. 2008;11:119-30.

40. Frye MA, Helleman G, McElroy SL, Altshuler LL, Black DO, Keck PE $\mathrm{Jr}$, et al. Correlates of treatment-emergent mania associated with antidepressant treatment in bipolar depression. Am J Psychiatry. 2009;166:164-72.

\section{Correspondence:}

Alisson Trevizol

Departamento de Psiquiatria, Faculdade de Ciências Médicas, Santa Casa de São Paulo

Rua Major Maragliano, 241, Vila Mariana

04017-030 - São Paulo, SP - Brazil

E-mail: alisson.trevizol@hotmail.com 